BULLETIN (New Series) OF THE

AMERICAN MATHEMATICAL SOCIETY

Volume 36, Number 4, Pages 413-432

S 0273-0979(99)00796-X

Article electronically published on July 21, 1999

\title{
LONGEST INCREASING SUBSEQUENCES: FROM PATIENCE SORTING TO THE BAIK-DEIFT-JOHANSSON THEOREM
}

\author{
DAVID ALDOUS AND PERSI DIACONIS
}

\begin{abstract}
We describe a simple one-person card game, patience sorting. Its analysis leads to a broad circle of ideas linking Young tableaux with the longest increasing subsequence of a random permutation via the Schensted correspondence. A recent highlight of this area is the work of Baik-Deift-Johansson which yields limiting probability laws via hard analysis of Toeplitz determinants.
\end{abstract}

\section{INTRODUCTION}

This survey paper treats two themes in parallel. One theme is a purely mathematical question: describe the asymptotic law (probability distribution) of the length of the longest increasing subsequence of a random permutation. This question has been studied by a variety of increasingly technically sophisticated methods over the last 30 years. We outline three, apparently quite unrelated, methods in sections 2, 3 and 4 . The other theme is a card game, patience sorting. This game provides an elementary context in which the longest increasing subsequence arises. More remarkably, it relates in different ways to two of the technical methods which have been used. Some new results concerning other aspects of patience sorting are described in section 5 .

1.1. Patience Sorting. One-person card games are called solitaire games in American and patience games in British. We start by describing one particular such game, patience sorting, invented as a practical method of sorting real decks of cards. History of this game and remarks on solitaire games which are traditionally played as recreation will be given later.

Take a deck of cards labeled $1,2,3, \ldots, n$. The deck is shuffled, cards are turned up one at a time and dealt into piles on the table, according to the rule

- A low card may be placed on a higher card (e.g. 2 may be placed on 7), or may be put into a new pile to the right of the existing piles.

At each stage we see the top card on each pile. If the turned up card is higher than the cards showing, then it must be put into a new pile to the right of the others. The object of the game is to finish with as few piles as possible.

To illustrate, suppose a shuffled deck of 10 cards is in the order

$$
\begin{array}{llllllllll}
7 & 2 & 8 & 1 & 3 & 4 & 10 & 6 & 9 & 5
\end{array}
$$

Received by the editors May 17, 1999.

1991 Mathematics Subject Classification. Primary 60C05, 05E10, 15A52, 60F05.

Research supported by NSF Grant MCS 96-22859.

(C)1999 American Mathematical Society 
The top card, 7 , is dealt face up on the table. The next card, 2, can be put on top of the 7 . But the 8 has to form a new pile, giving

\section{2 \\ 78}

The 1 can be placed on either the 2 or the 8 . If it were placed on the 8 , and if any of $\{3,4,5,6,7\}$ were to come next, the next card would have to form a new pile, whereas if the 1 were placed on the 2 , the 8 is still open for these cards. The greedy strategy is to always place a card on the leftmost possible pile. Adopting the greedy strategy (which we do unless otherwise stated) leads to the following sequence of configurations, where at each step, the top card in each pile is in boldface.

\begin{tabular}{|c|c|c|c|c|c|c|}
\hline 1 & 1 & 1 & 1 & 1 & 1 & 5 \\
\hline & 23 & 23 & 23 & $23 \quad 6$ & $23 \quad 6$ & 236 \\
\hline 8 & 78 & 784 & 78410 & 78410 & 784109 & 78410 \\
\hline
\end{tabular}

It is easy to see inductively that, under the greedy strategy, at each stage the labels on the top cards (i.e. the visible cards) are increasing from left to right. We mentioned that the object of the game is to finish with as few piles as possible. Lemma 1 will confirm one's intuition that the greedy algorithm is optimal.

To play with real cards one needs to linearly order the 52 cards, e.g. by putting suits in the bridge-bidding order $\diamond \nabla$. This mindless form of solitaire is then quite playable, perhaps while watching television.

It is natural to ask what a winning game should be. Monte Carlo simulations (see Table 1) show how the number of piles varies for an ordinary 52 -card deck.

$\begin{array}{rccccccccccc}\text { number of piles } & 8 & 9 & 10 & 11 & 12 & 13 & 14 & 15 & 16 & 17 & 18 \\ \text { frequency } & 54 & 525 & 1746 & 2791 & 2503 & 1518 & 632 & 186 & 33 & 11 & 1\end{array}$

TABLE 1. Number of final piles with $n=52$ and 10,000 trials.

The average number of piles is 11.6.

So usually there are 10 - 13 final piles, and we might count the solitaire game a success if we end with 9 piles or fewer. This leads to an approximate $5 \%$ chance of winning.

1.2. Patience sorting and longest increasing subsequences. A permutation $\pi$ of $\{1,2, \ldots, n\}$ can be identified with an arrangement of an $n$-card deck by specifying that $\pi(i)$ be the label of the card at position $i$. Thus for $n=10$ the arrangement displayed below is identified with the permutation $\pi(1)=7, \pi(2)=$ $2, \pi(3)=8, \ldots, \pi(10)=5$. An increasing subsequence $\left(i_{1}, i_{2}, \ldots, i_{k}\right)$ of $\pi$ is a subsequence satisfying

$$
i_{1}<i_{2}<\ldots<i_{k} ; \pi\left(i_{1}\right)<\pi\left(i_{2}\right)<\ldots<\pi\left(i_{k}\right) .
$$

For instance, the permutation

$$
\begin{array}{llllllllll}
7 & 2 & 8 & \underline{1} & \underline{3} & \underline{4} & 10 & \underline{6} & \underline{9} & 5
\end{array}
$$

has an increasing subsequence 
of length 5, and that is the longest possible for the permutation. We saw that patience sorting played with this permutation ended with 5 piles. This is no coincidence. Define $l(\pi)$ to be the length of the longest increasing subsequence of a permutation $\pi$.

Lemma 1. With deck $\pi$, patience sorting played with the greedy strategy ends with exactly $l(\pi)$ piles. Furthermore, the game played with any legal strategy ends with at least $l(\pi)$ piles. So the greedy strategy is optimal and cannot be improved by any look-ahead strategy.

Proof. If cards $a_{1}<a_{2}<\ldots<a_{l}$ appear in increasing order, then under any legal strategy each $a_{i}$ must be placed in some pile to the right of the pile containing $a_{i-1}$, because the card number on top of that pile can only decrease. Thus the final number of piles is at least $l$, and hence at least $l(\pi)$. Conversely, using the greedy strategy, when a card $c$ is placed in a pile other than the first pile, put a pointer from that card to the currently top card $c^{\prime}<c$ in the pile to the left. At the end of the game, let $a_{l}$ be the card on top of the rightmost pile $l$. The sequence

$$
a_{1} \leftarrow a_{2} \leftarrow \ldots \leftarrow a_{l-1} \leftarrow a_{l}
$$

obtained by following the pointers is an increasing subsequence whose length is the number of piles.

In the example, the pointers look like

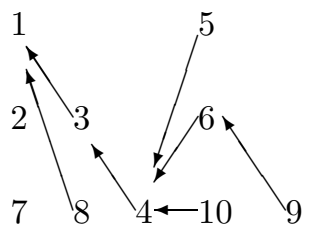

and we recover the subsequence 13469 of (1). Note that the final top cards are in general not an increasing subsequence: in the example, 5 came after 9 in the deck. Note also that the maximal subsequence is in general not unique: we could take " 2 " in place of " 1 " in (1).

1.3. Other appearances of $l(\pi)$. Thinking of a permutation $\pi$ as an arrangement of a deck of cards, a natural metric $U\left(\pi_{1}, \pi_{2}\right)$ on permutations can be defined as follows. Say a move consists of moving a single card to some other position within the deck; then $U\left(\pi_{1}, \pi_{2}\right)$ is the minimum number of moves required to go from arrangement $\pi_{1}$ to arrangement $\pi_{2}$. More mathematically, for any set $G$ of generators of the symmetric group $S_{n}$, we can define a distance or length function $d\left(\pi_{1}, \pi_{2}\right)$ to be

$$
\min \left\{d: \pi_{1} g_{1} g_{2} \ldots g_{d}=\pi_{2} \text { for some } g_{i} \in G\right\} .
$$

Then $U\left(\pi_{1}, \pi_{2}\right)$ is the distance associated with the generators $\left\{c_{i j}^{ \pm 1}, 1 \leq i<j \leq n\right\}$, where $c_{i j}$ is the cycle $(i, i+1, \ldots, j)$. It is not hard to show that, writing $\iota$ for the identity permutation,

$$
U(\iota, \pi)=n-l(\pi)
$$

This $U$ is Ulam's metric [10], [53]. Ulam (personal communication) relates that he thought of this metric while thinking about the fastest way to sort a bridge hand of thirteen cards. 
Note that the definition of $l(\pi)$ makes its computation look like a problem in combinatorial optimization, a field in which many problems are algorithmically hard. But Lemma 1 shows that patience sorting is implicitly an algorithm for computing $l(\pi)$ and exhibiting a maximal-length increasing subsequence. Fredman [16] has shown this algorithm can be implemented using $n \log n-n \log \log n+$ $O(n)$ comparisons in the worst case, and that no algorithm has better worst-case behavior.

1.4. The behavior of $l(\pi)$ for large $n$. Patience sorting and Ulam's metric provide two elementary motivations for studying $l(\pi)$, and more sophisticated motivations arise from the connections to be described below. We seek to study the "typical" values of $l(\pi)$ when $n$ is large. In the language of probability theory, we take a uniform random permutation $\pi_{n}$ of $\{1,2, \ldots, n\}$ and study the law of the integer-valued random variable $L_{n}:=l\left(\pi_{n}\right)$, which can be summarized by its mean $E\left[L_{n}\right]$ and standard deviation $\sigma\left[L_{n}\right]$. (Probabilists usually write about the variance $\sigma^{2}$ instead of the standard deviation $\sigma$, but the latter is the intuitively interpretable measure of spread.)

A fundamental theme of this survey is that there are three distinct ways to relate $L_{n}$ to some apparently quite different objects.

- The Schensted correspondence (section 2.1) gives a formula (3) for $P\left(L_{n}=l\right.$ ) in terms of the shape of random Young tableaux.

- Corollary 5 in section 3.2 represents $L_{n}$ as the number of particles at time $n$ in a certain interacting particle system on the real line.

- Proposition 6 in section 4 gives a formula for $P\left(L_{n} \leq l\right)$ in terms of an integral with respect to Haar measure on the unitary group, and Proposition 7 reinterprets this in terms of a Toeplitz determinant.

In each case it is possible (though not easy) to perform an asymptotic analysis of the related structure and derive information about the asymptotic behavior of $L_{n}$. We shall outline these relations and the analysis in sections 2, 3 and 4, but here is a summary. Hammersley [19] gave the first extensive discussion, and then independently Vershik and Kerov [55] and Logan and Shepp [29] gave results showing essentially

Theorem 2. As $n \rightarrow \infty$ we have $E\left[L_{n}\right] \sim 2 n^{1 / 2}$ and $\sigma\left[L_{n}\right]=o\left(n^{1 / 2}\right)$.

Subsequently it was conjectured - we first heard the conjecture from Kesten (personal communication), inspired by analogous conjectures in first passage percolation ([25], page 298) and Hammersley's representation (6), and endorsed by Monte Carlo simulation (table 2) - that more detailed asymptotics were

$$
E\left[L_{n}\right]=2 n^{1 / 2}-\mu_{\infty} n^{1 / 6}+o\left(n^{1 / 6}\right), \quad \sigma\left[L_{n}\right]=\sigma_{\infty} n^{1 / 6}+o\left(n^{1 / 6}\right)
$$

for constants $\mu_{\infty}$ and $\sigma_{\infty}$. Kim [26] proved the $O\left(n^{1 / 6}\right)$ bound on the upper tail of the distribution of $L_{n}$, and very recent work of Baik-Deift-Johansson [3] gives a

$\begin{array}{rccccccc}n & 10^{4} & 10^{5} & 10^{6} & 10^{7} & 10^{8} & 10^{9} & 10^{10} \\ \text { sample size } & 10^{7} & 6 \cdot 10^{5} & 10^{5} & 10^{5} & 10^{4} & 2000 & 4000 \\ \text { estimated } \mu_{\infty} & 1.660 & 1.697 & 1.720 & 1.733 & 1.738 & 1.735 & 1.774 \\ \text { estimated } \sigma_{\infty} & 0.871 & 0.885 & 0.896 & 0.900 & 0.898 & 0.902 & 0.895\end{array}$

TABLE 2. Monte Carlo simulation results by Odlyzko and Rains [37], giving for each $n$ the values of these constants estimated from the samples. 
rigorous proof of (2) with numerical values $\mu_{\infty}=1.711 \ldots, \sigma_{\infty}=0.902 \ldots$, and also provides the asymptotic law, to which we shall return in section 4.1. The asymptotic approximation (2) is quite accurate for $n=52$, predicting a value 11.12 for $E\left[L_{52}\right]$ where the simulation in table 1 gave 11.6.

1.5. History of patience sorting. The name patience sorting comes from Mallows [32], who in [31] credits A.S.C. Ross for its invention with the following motivation. At the end of the game, card 1 is always on top of the leftmost pile. Removing card 1, card 2 is now at the top of some pile; removing card 2, card 3 is now at the top of some pile, and so we have a natural algorithm for manually sorting cards. Whether this is the fastest practical method for sorting real cards (or alphabetizing final exams) is an interesting topic for coffee-room conversation.

Mallows' analysis [32] was done in 1960 but not published until much later (see section 2.3 for more remarks). Independently, patience sorting was discovered by computer scientist Bob Floyd in 1964 and developed briefly in letters between Floyd and Knuth, but their work apparently has never been published. Hammersley ([19], p. 362) independently recognized its use an an algorithm for computing $l(\pi)$.

\section{The Young tableaux method}

The Schensted correspondence is a remarkable bijection between permutations and pairs of standard Young tableaux. It was invented by Schensted [45] to study the length of the longest increasing subsequence. We have found that the solitaire game of section 1.1 gives an easy, clear way of explaining the correspondence. In section 2.1 we give background on Young tableaux. In section 2.2 we show how one can use the correspondence to get asymptotic information about $L_{n}$.

2.1. Young tableaux and the Schensted correspondence. Here we state some standard facts taken from the undergraduate textbook Stanton and White [49], secs. 3.5-3.7. More sophisticated treatments, emphasizing representation theory for the symmetric group, can be found in Sagan [44], MacDonald [30], James and Kerber [20], Stanley [47].

A partition $\lambda=\left(\lambda_{1}, \ldots, \lambda_{j}\right)$ of an integer $n \geq 1$ is a sequence with $\lambda_{1} \geq \lambda_{2} \geq$ $\ldots \geq 1$ and $\sum_{i} \lambda_{i}=n$. We may identify a partition $\lambda$ with its associated Ferrers diagram of $n$ cells with $\lambda_{i}$ cells in row $i$, drawn as shown below. The hook length $h_{c}$ of a cell $c$ in a Ferrers diagram is the number of cells in the set consisting of the cells to the right of $c$ in its row, the cells below $c$ in its column, and cell $c$ itself.

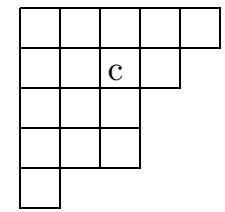

$$
\begin{aligned}
& \lambda=(5,4,3,3,1) \\
& h_{c}=4
\end{aligned}
$$

A (standard) Young tableau of shape $\lambda$ is a Ferrers diagram with the cells occupied by the numbers $1,2, \ldots, n$ in such a way that the numbers increase along each row and down each column. Examples are in Figure 1. The number of Young tableaux of a given shape $\lambda$ is denoted by $d_{\lambda}$ and is given by the hook formula of Frame Robinson: see Stanton and White [49], Theorem 5.4. 


\begin{tabular}{llllllllll}
418 & \multicolumn{8}{c}{ DAVID ALDOUS AND PERSI DIACONIS } \\
& 2 & 28 & 18 & 13 & 134 & 13410 & 1346 & 13469 & 13459 \\
$\mathrm{P}$ & 7 & 7 & 2 & 28 & 28 & 28 & 2810 & 2810 & 2610 \\
& & & 7 & 7 & 7 & 7 & 7 & 7 & 78 \\
& 1 & 13 & 13 & 13 & 136 & 1367 & 1367 & 13679 & 13679 \\
$\mathrm{Q}$ & 2 & 2 & 2 & 25 & 25 & 25 & 258 & 258 & 258 \\
& & & 4 & 4 & 4 & 4 & 4 & 4 & 410
\end{tabular}

Figure 1. Constructing the Schensted correspondence for the permutation $\begin{array}{llllllllll}7 & 2 & 8 & 1 & 3 & 4 & 10 & 6 & 9 & 5\end{array}$

Lemma 3 (The hook formula).

$$
d_{\lambda}=\frac{n !}{\prod_{c} h_{c}} .
$$

The Schensted (or Robinson-Schensted-Knuth) correspondence is a bijection between permutations of $n$ cards and ordered pairs of Young tableaux of size $n$ with the same (unspecified) shape $\lambda$. The existence of such a bijection implies the formula

$$
n !=\sum_{\lambda \vdash n} d_{\lambda}^{2}
$$

where $\lambda \vdash n$ is an abbreviation for " $\lambda$ is a partition of $n$ ".

The two tableaux are called the $P$-tableau and the $Q$-tableau. As with patience sorting, the correspondence can be constructed inductively by specifying where to place the next card. Roughly speaking, a tableau is constructed by a recursive use of patience sorting. When a card is covered, it is moved again according to patience sorting applied to the cards at the second level, and so on. In more detail:

Place the next card in the top row of the $P$-tableau, in the position of the smallest higher-numbered card if any exists (thereby "bumping" that card), or append to the right end of the top row if no such highernumbered card exists. If a card is bumped, consider the $P$-tableau with top row deleted, and recursively use the same rule to insert the bumped card into the remaining tableau. This eventually yields a new $P$-tableau whose shape is the previous tableau with one extra cell. Make the new $Q$-tableau be the previous $Q$-tableau with this extra cell and with the number $m$ in that cell, where the added card is the $m$ 'th card dealt.

Figure 1 illustrates the construction on our running example. It is not hard to see that the correspondence can be worked backwards. Thus from the final $P$ and $Q$ in the example above, the position of 10 in the $Q$-tableau indicated where the last box was added. Looking at the $P$-tableau, one can see that this happened by the 6 bumping the 8 , which was caused by the 5 bumping the 6 , so $\pi(10)=5$.

Note in particular

- The top row in the $P$-tableau is the same as the row of top cards in patience sorting.

So the length of the top row in the Young tableau associated with a permutation $\pi$ is the length $l(\pi)$ of the longest increasing subsequence of $\pi$. The existence of the bijection now allows us to write a formula for the probability $P\left(L_{n}=l\right)$ that the 
longest increasing sequence of a uniform random permutation of $\{1,2, \ldots, n\}$ has length $l$.

\section{Proposition 4.}

$$
P\left(L_{n}=l\right)=\frac{1}{n !} \sum_{\substack{\lambda \vdash n \\ \lambda_{1}=l}} d_{\lambda}^{2}
$$

2.2. Analysis of the asymptotics. To get asymptotics for $L_{n}$ from the Schensted correspondence, we consider, instead of just the top row, the shape of the whole tableau. Let $\Lambda^{(n)}=\left(\Lambda_{i}^{(n)} ; i \geq 1\right)$ be a (non-uniform) random partition of $n$ with distribution

$$
P\left(\Lambda^{(n)}=\lambda\right):=d_{\lambda}^{2} / n !=n ! /\left(\prod_{c} h_{c}\right)^{2}
$$

corresponding to the hook formula (Lemma 3). Though the shapes (and so the probabilities) of different partitions vary widely, it is natural to guess (by analogy with classical results in statistical physics or "large deviations" in probability theory) that for large $n$ the distribution is concentrated near the most likely shape. It is easy to outline an asymptotic analysis which confirms this. The hook length for a cell $(i, j)$ (the $j$ 'th cell in row $i$ ) in a partition $\lambda=\left(\lambda_{i}\right)$ has

$$
h_{(i, j)} \approx \lambda_{i}-j+\lambda_{j}^{-1}-i
$$

where $\lambda^{-1}$ denotes the inverse function and where $\approx$ indicates a heuristic approximation. Rescale the row-lengths of $\lambda$ to define

$$
z_{\lambda}(x)=n^{-1 / 2} \lambda_{\left\lfloor n^{1 / 2} x\right\rfloor} ; \quad x \geq 0 .
$$

Then

$$
\log \prod_{c} h_{c} \approx \frac{1}{2} n \log n+n H\left(z_{\lambda}\right)
$$

where

$$
H(f):=\int_{0}^{\infty} \int_{0}^{f(x)} \log \left(f(x)-y+f^{-1}(y)-x\right) d y d x
$$

This leads to

$$
P\left(\Lambda^{(n)}=\lambda\right) \approx \exp \left(-2 n\left(H\left(z_{\lambda}\right)+\frac{1}{2}+o(1)\right)\right) .
$$

The approximation (4) suggests that the random tableaux $\Lambda^{(n)}$ do have a limit deterministic shape, more precisely, that the random process $Z_{n}(x)=n^{-1 / 2} \Lambda_{\left\lfloor n^{1 / 2} x\right\rfloor}^{(n)}$ of rescaled row-lengths converges to the function $f_{*}(x)$ which solves the calculus of variations problem

minimize $H(f)$ over nonincreasing $f:(0, \infty) \rightarrow[0, \infty)$ with $\int_{0}^{\infty} f(x) d x=1$.

It turns out that the minimizing function $f_{*}(x)$ is zero outside $[0,2]$, and on $[0,2]$ is specified via

$$
f_{*}(x)=2 \pi^{-1}(\sin \theta-\theta \cos \theta), x=f_{*}(x)+2 \cos \theta, \quad 0 \leq \theta \leq \pi .
$$

In particular, $f_{*}(0)=2$. Since $L_{n}$ is the length of the top row of the tableau associated with $\Lambda^{(n)}$, one is led to believe

$$
n^{-1 / 2} L_{n}=Z_{n}(0) \rightarrow f_{*}(0)=2 .
$$


This argument was developed rigorously by Logan and Shepp [29], who used it to conclude

$$
E\left[L_{n}\right] \geq(2+o(1)) n^{1 / 2} .
$$

Vershik and Kerov [55], [24], [56] independently outlined the same argument for this lower bound and an argument for the upper bound $E\left[L_{n}\right] \leq(2+o(1)) n^{1 / 2}$. This provided the first proof of Theorem 2. Subsequently it was proved in Pilpel [40] that in fact $E\left[L_{n}\right] \leq 2 n^{1 / 2}$ for all $n$; we have also seen an (apparently unpublished) simple proof of this by Verskik - Kerov.

2.3. More history of patience sorting. Mallows [32] records how he discovered that the number of piles in patience sorting relates to Young tableaux. Barton [31] pointed out the connection with the Schensted correspondence. Using these, Mallows proved that the chance that patience sorting yields $l$ piles and also yields $r$ piles when played with the deck in reversed order equals the sum (3) further restricted to tableaux $\lambda$ with $r$ rows.

2.4. Patience sorting for decks with repeated values. In playing patience sorting with real cards we could regard all cards of the same rank (e.g. all four 8's) as equal. We then have two possible rules for playing: either ties allowed, e.g. an 8 may be played on top of another 8 , or ties forbidden. Let us indicate how the "ties forbidden" game permits a development related to the Schensted correspondence.

Mathematically, consider an $n$-card deck with $a_{i}$ cards labeled $i$, for $1 \leq i \leq A$. For instance, a deck with $n=9$ and $a_{1}=a_{2}=a_{3}=3$ might be in order

\section{$\underline{2} \underline{3} 12 \underline{3} 121 \underline{3}$}

Playing with ties forbidden results in the final configuration

122

2333

There are four piles, and one can check that in the original deck the longest weakly increasing subsequence has length four. This connection is general and, analogously to permutations, gives a bijection between the $\left(\begin{array}{c}n \\ a_{1} \ldots a_{A}\end{array}\right)$ deck orders and pairs $(P, Q)$ of Young tableaux of the same shape $\lambda$ with $Q$ standard and $P$ semi-standard (that is, rows are weakly increasing and columns are strictly increasing, the entries being $a_{i}$ repeats of $i$ ). The number of cells in the first row of the common shape $\lambda$ is the length of the longest weakly increasing subsequence. For the deck order above,

$$
\begin{aligned}
& 1113 \quad 1259 \\
& \begin{array}{lll} 
& 222 & \mathrm{Q} \quad 347
\end{array} \\
& 33 \quad 68
\end{aligned}
$$

Asymptotics for general $\left(a_{i}\right)$ have not been studied. However, the special case $n=k, a_{1}=a_{2}=k / 2$ (for even $k$ ) is implicit in classical probability. Write

$$
M_{k}(i)=(\text { number of 1's in positions } 1 \text { through } i)-\frac{i}{2} .
$$

Because a weakly increasing subsequence consists of the 1's up to some position $i$ and the 2's thereafter, it is easy to see that the length $L_{k}$ of the longest weakly 
increasing subsequence is

$$
L_{k}=\frac{k}{2}+2 \max _{i} M_{k}(i)
$$

So as $k \rightarrow \infty$

$$
P\left(L_{k} \leq \frac{k}{2}+2 k^{1 / 2} x\right)=P\left(k^{-1 / 2} \max _{i} M_{k}(i) \leq x\right) \rightarrow 1-e^{-2 x^{2}}
$$

by classical results on weak convergence of sampling processes to Brownian bridge ([7], (11.40) and Theorem 24.1). More sophisticated results on analogous problems are mentioned at the end of section 4.1.

Remark. One could also analyze the "ties allowed" game in simple cases. But there is no bijection between this (or any other solitaire game we know) and pairs of Young tableaux with both tableaux semi-standard.

\section{Probabilistic methods}

3.1. Subadditivity. Hammersley [19] gave a representation of $L_{n}$ in terms of a Poisson random process of points in the quadrant $[0, \infty)^{2}$; see $(6)$. The purpose of that representation is a superadditivity property which easily implies $E\left[L_{n}\right] \sim$ $c n^{1 / 2}$ for some unspecified $c$. Hammersley's construction is nowadays a textbook application ([13], Example 6.7.2) of the subadditive ergodic theorem, which in our context implies

$$
P\left(\left|n^{-1 / 2} L_{n}-c\right|>\varepsilon\right) \rightarrow 0, \varepsilon>0 .
$$

But these methods do not directly specify the value of $c$, which from Theorem 2 we know equals 2 .

3.2. An interacting particle process. Instead of thinking of the cards in an $n$-card deck being labeled $\{1,2, \ldots, n\}$ and shuffled into random order, we can equivalently think of the labels on successive cards being independent random real numbers, uniformly distributed on $[0,1]$. The point of this reformulation is that we can compare configurations as $n$ varies. For each $n$ consider the labels on the top card of each pile after $n$ cards have been played, and interpret these labels as the positions of "particles" on $[0,1]$. The rule for playing patience sorting becomes the following rule for the evolution of an interacting particle process. Initially there are zero particles.

At each step, a particle appears at a uniform random point $U$ in $[0,1]$; simultaneously the nearest particle (if any) to the right of $U$ disappears.

So Lemma 1 implies

Corollary 5. The number of particles after $n$ steps is distributed as $L_{n}$.

Incidently, Corollary 5 provides a fairly efficient algorithm for simulating $L_{n}$.

Having switched from discrete to continuous labels, we next switch from discrete to continuous time. That is, we think of new particles appearing at the time of a Poisson process of rate 1: informally, in a time interval $[t, t+d t]$ a new particle appears with chance $d t$. In the rule above we spoke of a particle appearing and another simultaneously disappearing, but it is natural to rephrase this in terms of a particle instantaneously changing position. This rephrasing gives the following evolution rule for a particle process in continuous time and space.

For each interval $[x, x+d x]$ at time $t$, with probability $d x d t$ the nearest particle to the right of $x$ is moved to $x$ by time $t+d t$. 


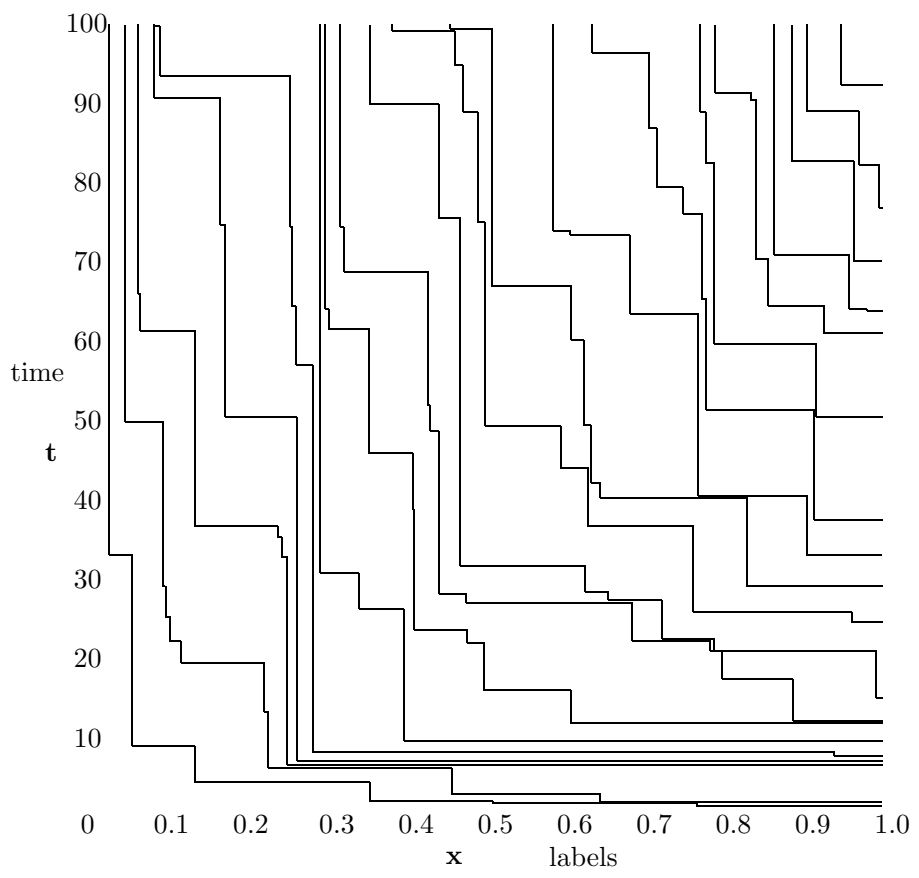

FiguRE 2. Labels on the top cards of each pile in patience sorting, in continuized time

Instead of taking the spatial interval to be $[0,1]$ we can take it to be $[0, \infty)$; somewhat paradoxically there is a unique way to start the process with zero particles at time zero but infinitely many particles at each positive time. We call this Hammersley's process (though it's far from explicit in [19]), and write $\hat{L}(x, t)$ for the number of particles at time $t$ in the interval $[0, x]$. Figure 2 is a Monte Carlo simulation, in which each path shows the position of a given particle as time increases (vertical scale), or equivalently the label of the top card in a given pile in patience sorting after switching to continuous time. The exact connection with $L_{n}$ is via Poissonization:

$$
\hat{L}(1, t)=L_{N(t)}, \text { where } N(t) \text { has } \operatorname{Poisson}(t) \text { law, }
$$

that is,

$$
P(N(t)=n)=e^{-t} t^{n} / n !, n=0,1,2, \ldots
$$

Remark. The connection with Hammersley's superadditive representation is as follows. Underlying the evolution rule is a space-time Poisson process (of rate 1) of random points $\left(x^{\prime}, t^{\prime}\right)$ in the quadrant $[0, \infty)^{2}$. One can check

$$
\begin{aligned}
\hat{L}(x, t)= & \text { maximal number of such points } \\
& \text { on any up-right path from }(0,0) \text { to }(x, t) .
\end{aligned}
$$

(An up-right path being one that can be written as $((x(u), t(u)), u \geq 0)$ for nondecreasing $x(\cdot)$ and $t(\cdot))$. This presents the $L_{n}$-problem as a continuous-space analog 
of first passage oriented percolation [12], an analogy that was natural to Hammersley as a founder of the mathematical theory of percolation [18].

3.3. Analysis of the asymptotics. Aldous and Diaconis [1] used the interacting particle representation to re-prove Theorem 2. Here is an outline of the argument.

Suppose the spatial process around position $x$ at time $t$ approximates a Poisson process of some rate $\lambda(x, t)$. Clearly

$$
\frac{d}{d t} E \hat{L}(x, t)=E D_{x, t}
$$

where $D_{x, t}$ is the distance from $x$ to the nearest particle to the left of $x$. For a Poisson process $E D_{x, t}$ would be 1/ (spatial rate), so

$$
E D_{x, t} \approx \frac{1}{\lambda(x, t)} \approx \frac{1}{\frac{d}{d x} E \hat{L}(x, t)} .
$$

In other words, $w(x, t)=E \hat{L}(x, t)$ satisfies approximately the PDE

$$
\frac{d w}{d t}=\frac{1}{\frac{d w}{d x}} ; \quad w(0, x)=w(t, 0)=0
$$

whose solution is

$$
w(x, t)=2 \sqrt{t x} .
$$

Note that " 2 " is not an arbitrary constant: no other constant will serve. In other words,

$$
E\left[L_{n}\right] \approx E \hat{L}(1, n)=w(n, 1) \approx 2 n^{1 / 2} .
$$

In the language of statistical physics, what we have outlined is a hydrodynamic limit argument. See de Masi et al. [11] for a survey. The analysis of Hammersley's process given in [1] closely parallels the earlier analysis of the (discrete space, continuous time) simple asymmetric exclusion process in one dimension, due to Rost [43]; see also Liggett [28], sec. 8.5. Subsequently, Venkatsubramani [54] studied the analog of Hammersley's process on the circle, and Seppäläinen [46] developed the hydrodynamical argument of [1] in a more abstract setting where it can be applied to other growth models.

\section{The RANDOM MATRIX CONNECTION}

The most recent and deepest study of $L_{n}$ exploits a connection with random matrices, based on the following formula discovered by Rains [42] and Odlyzko et al. [36].

\section{Proposition 6.}

$$
P\left(L_{n} \leq l\right)=\frac{1}{n !} \int_{U_{l}}|\operatorname{Tr} M|^{2 n} d M .
$$

Here $d M$ denotes normalized Haar measure on the unitary group $U_{l}$ of $l \times l$ matrices $M$.

This result is surprising but not difficult to see once someone tells you it is true. The average of the trace is the inner product

$$
\int_{U_{l}}|\operatorname{Tr} M|^{2 n} d M=\left\langle P_{1}^{n} \mid P_{1}^{n}\right\rangle
$$


where $P_{1}\left(x_{1}, \ldots, x_{l}\right)=\sum_{i=1}^{l} x_{i}$ is the first power sum symmetric function applied to the eigenvalues of $M$. Any symmetric homogeneous polynomial of degree $n$ in $l$ variables can be expressed as a linear combination of Schur functions. For $P_{1}^{n}$ this was done by Frobenius. The result is

$$
P_{1}^{n}=\sum_{\mu \vdash l} \chi_{\mu}\left(1^{l}\right) s_{\mu}
$$

where $\left\{s_{\mu}: \mu \vdash n\right\}$ are the Schur functions, which are the characters of the unitary group $U_{l}$. The $s_{\mu}$ satisfy $\left\langle s_{\mu} \mid s_{\nu}\right\rangle=\delta_{\mu \nu}$ and $s_{\mu}=0$ if the conjugate partition $\mu^{T}$ has $\mu_{1}^{T}>l$. The numbers $\chi_{\mu}(\lambda)$ are the characters of the symmetric group. In the application $\lambda=1^{l}$, and so $\chi_{\mu}(\lambda)=d_{\mu}$ is the dimension of the $\mu^{\prime}$ th irreducible representation. Putting these pieces together gives

$$
\int_{U_{l}}|\operatorname{Tr} M|^{2 n} d M=\sum_{\mu \vdash n, \mu_{1}^{T} \leq l}\left(\chi_{\mu}\left(1^{l}\right)\right)^{2} .
$$

Using the Schensted correspondence (Proposition 4 above), we see the sum equals the number of permutations $\pi$ of $\{1, \ldots, n\}$ with $l(\pi) \leq l$. Dividing both sides by $n$ ! establishes (9).

From the definition of Haar measure we can rewrite the right side of (9) as

$$
\frac{1}{n !(2 \pi)^{l}} \int_{0}^{2 \pi} \ldots \int_{0}^{2 \pi}\left|e^{i \theta_{1}}+\ldots+e^{i \theta_{l}}\right|^{2 n} \prod_{1 \leq j<k \leq l}\left|e^{i \theta_{j}}-e^{i \theta_{k}}\right|^{2} d \theta_{1} \ldots d \theta_{l} .
$$

The next step is to relate (10) to a formula involving Toeplitz determinants. For a function $f(\theta)$ on the unit circle with Fourier coefficients $\hat{f}(j)$, we can construct an $l \times l$ Toeplitz matrix with entries $(\hat{f}(k-j), 1 \leq j, k \leq l)$. Write $D_{l-1}(f(\cdot))$ for its determinant. A classical identity due to Heine says

$$
\frac{1}{(2 \pi)^{l} l !} \int \ldots \int f\left(\theta_{1}\right) \ldots f\left(\theta_{l}\right) \prod_{1 \leq j<k \leq l}\left|e^{i \theta_{j}}-e^{i \theta_{k}}\right|^{2} d \theta_{1} \ldots d \theta_{l}=D_{l-1}(f(\cdot)) .
$$

Comparing this with (10), we see we have an expression for the distribution of the Poissonized quantity $L_{N(t)}$ at (5).

\section{Proposition 7.}

$$
P\left(L_{N(t)} \leq l\right)=\sum_{n} \frac{e^{-t} t^{n}}{n !} P\left(L_{n} \leq l\right)=e^{-t} D_{l-1}\left(e^{2 \sqrt{t} \cos }\right) .
$$

This identity can be seen as a special case of a result of Gessel [17]. Incidently, the density $\exp (\alpha \cos (\cdot))$ on the circle is the classical von Mises - Fisher or circular Normal distribution ([23], Chapter 33).

4.1. Asymptotic analysis. Over the past 50 years there has been ongoing study of the asymptotics of Toeplitz determinants. To study the asymptotic law of $L_{n}$ via (11), one needs two-parameter asymptotics, since $t$ is of order $n$ and $l$ is of order $n^{1 / 2}$. Baik-Deift-Johansson [3] combine existing methods with some profound new analysis to show 
Theorem 8. Let $q(x)$ be the Painlevé II function solving $q^{\prime \prime}(x)=x q(x)+2 q^{3}(x)$ for which $q(x) \sim \operatorname{Ai}(x)$ as $x \rightarrow \infty$, where Ai is Airy's function. Write

$$
F(x)=\exp \left(-\int_{x}^{\infty}(x-t) q^{2}(t) d t\right)
$$

Then

$$
P\left(\frac{L_{n}-2 n^{1 / 2}}{n^{1 / 6}} \leq x\right) \rightarrow F(x) \text { as } n \rightarrow \infty,-\infty<x<\infty .
$$

Remarkably, the same limit law arose earlier. Tracy and Widom [50] studied the size of the largest eigenvalue ( $\lambda_{n}^{*}$, say) of a random $n \times n$ Hermitian matrix and obtained the result of Theorem 8 with $\lambda_{n}^{*}$ in place of $L_{n}$. It is unknown whether there is some "finite $n$ " identity which explains this coincidence.

The Baik-Deift-Johansson theorem has set off an avalanche of research activity. Tracy and Widom [51] relate Theorem 8 to the existing results on random matrices and the appearance of Painlevé functions. Borodin [8] used it to prove similar results for wreath products. Johansson [22] finds the same limit law $F(x)$ in the following discrete analog of $(6)$ : set independent random variables with geometric distribution on the vertices of the grid $[0, n] \times[0, n]$, and let $M_{n}$ be the maximum, over upright paths from $(0,0)$ to $(n, n)$, of the sum of the variables on the path. Baik and Rains [6], [5] have shown that much of the machinery carries over to subsets of permutations, such as involutions, and have given combinatorial proofs of apparently purely analytic equivalences.

One of the key issues is seeing sharply why there should be a connection between permutations and random matrices. Okounkov [38] gives a surprising connection via Riemann surfaces. The set of triangulations of a surface can be described through integrals over matrix ensembles using work of Kontsevich. Permutations enter in describing the monodromy of simply branched covers. Okounkov used these ideas to show that the limiting distribution for the number of cells in the $i$ 'th row of the Schensted tableau agrees with the limiting distribution for the $i$ 'th largest eigenvalue of a random Hermitian matrix. The latter distribution (different from $F(x)$ ) was earlier determined by Tracy and Widom [50]. A different proof of this asymptotic equidistribution is announced in Borodin et al. [9] (and for $i=2$ given by Baik-Deift-Johansson [4]).

Somewhat related to random permutations of an $n$-set is the study of uniform random words of length $n$, with letters from $\{1,2, \ldots, k\}$. Tracy and Widom [52] study the length $L_{n, k}$ of the longest weakly increasing subsequence and show

$$
P\left(\frac{L_{n, k}-n / k}{n^{1 / 2}} \leq x\right) \rightarrow P\left(\lambda_{\max }^{(k)} \leq x \sqrt{k / 2}\right) \text { as } n \rightarrow \infty \text { for fixed } k
$$

where $\lambda_{\max }^{(k)}$ is the largest eigenvalue for a random matrix in the Hermite ensemble of $k \times k$ traceless Hermitian matrices. It is intriguing to speculate that there may be connections with the subject of sequence comparison statistics, motivated by DNA sequence matching (e.g. Waterman and Vingron [57]), where analogous asymptotic distribution theory is less well developed.

\section{More about PATIEnCE SORTing}

From the viewpoint of patience sorting with $n$ cards, the number of piles is just one aspect of the final configuration. There are other natural questions, such as 


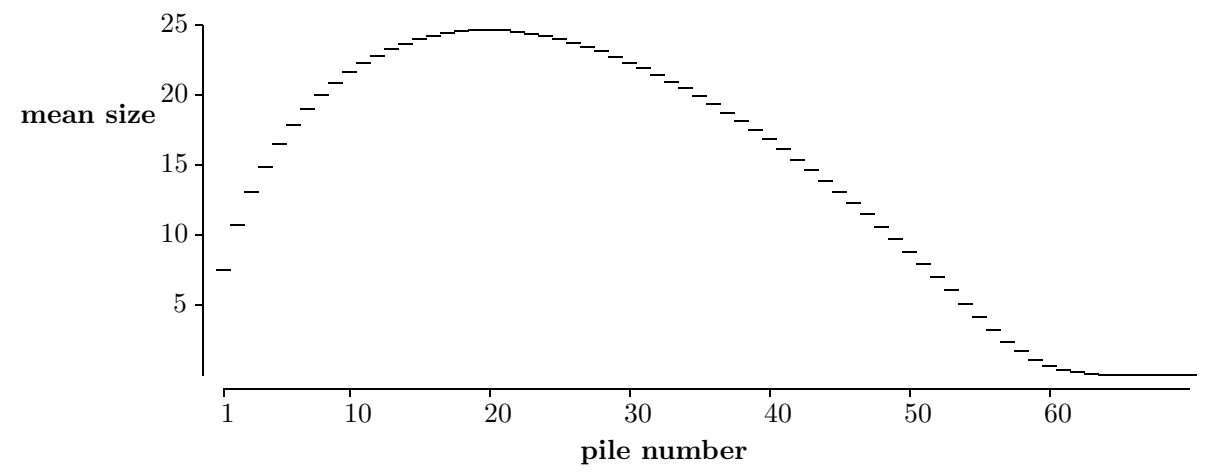

Figure 3. Mean pile sizes for $n=1000$, estimated from $10^{4}$ Monte Carlo trials

the number of cards in the different piles. These questions have not been studied explicitly in the literature, but as the following outline shows, their analysis also relates to diverse topics in probability and combinatorics. Details may be published elsewhere.

Simulations (see figure 3) reveal a definite shape for pile sizes, for large $n$. We shall outline separate analyses for the leftmost piles, the central piles and the rightmost piles.

5.1. The first pile. The cards which enter the first pile are exactly the record cards, i.e. those whose label is smaller than any previous card. Thus in our example$$
\begin{array}{llllllllll}
\underline{2} & \underline{2} & 8 & \underline{1} & 3 & 4 & 10 & 6 & 9 & 5
\end{array}
$$

the records are $7,2,1$. Writing $A_{i}$ for the event "the $i$ 'th card is a record", it is elementary that $P\left(A_{i}\right)=1 / i$ and that the events $\left(A_{1}, \ldots, A_{n}\right)$ are independent. Thus $S_{n}(1)$, the total number of records in a random permutation of $n$ cards (and the number of cards in pile 1 in patience sorting), satisfies

$$
\begin{gathered}
E S_{n}(1)=\sum_{i=1}^{n} \frac{1}{i} \sim \log n \\
\sigma\left[S_{n}(1)\right]=\sum_{i=1}^{n} \frac{1}{i}\left(1-\frac{1}{i}\right) \sim \log n .
\end{gathered}
$$

And a textbook application (e.g. [13], p. 102) of the central limit theorem for independent non-identically distributed random variables shows

$$
P\left(\frac{S_{n}(1)-\log n}{\sqrt{\log n}} \leq x\right) \rightarrow \Phi(x),-\infty<x<\infty
$$

for $\Phi(x):=\int_{-\infty}^{x}(2 \pi)^{-1 / 2} e^{-y^{2} / 2} d y$.

We remark that records form a well studied classical topic in probability theory: see e.g. Arnold et al. [2]. There are connections with group theory, for instance the joint distribution of inter-record times is the joint distribution of cycle lengths in a uniform random permutation ([48], Chapter 1). 
5.2. The leftmost piles. We can study the analogous questions for $S_{n}(i)$, the number of cards in pile $i$. In a certain sense the cards in pile $i$ are " $i$ 'th records": if the record values in a random permutation are deleted, the 2 'nd records are the record times amongst the remaining values, and so on. Note this interpretation of $i$ 'th record is different from the more elementary interpretation (a card is an $i$ 'th record if exactly $i-1$ earlier cards had lower rank) used in the remarkable Ignatov's theorem [14], which asserts a certain independence property in the latter context.

It is not hard to relate the expectation $E S_{n}(i)$ to the distributions of the $L_{n}$.

Proposition 9. For $n, i \geq 1$

$$
E S_{n}(i)=\sum_{k=1}^{n} P\left(L_{k-1}=i-1\right) \sum_{j=k}^{n} \frac{1}{j}
$$

where we interpret $L_{0}$ as 0 . For fixed $i \geq 1$,

$$
E S_{n}(i)=c(i) \log n+O(1) \text { as } n \rightarrow \infty
$$

where

$$
c(i):=\sum_{k=i}^{\infty} P\left(L_{k-1}=i-1\right)<\infty .
$$

And $c(1)=1, \quad c(2)=e-1$,

$$
c(3)=\sum_{k=1}^{\infty} \frac{1}{k !}\left(\left(\begin{array}{c}
2 k \\
k
\end{array}\right) \frac{1}{k+1}-1\right) \approx 2.3724 .
$$

We do not have a useful closed-form expression for the general $c(i)$, though an implicit formula follows from (3) and the definition of $c(i)$.

Proposition 9 is a "combinatorial" result based upon exact formulas. In contrast, we can extend the central limit theorem (13) by soft "probabilistic" arguments. Recall the interacting particle process setup of section 3.2. By using a different rescaling of labels, for fixed $k$ we can define a $k$-particle process representing rescaled labels of the top cards on the first $k$ piles. This turns out to be a continuous-time Harris positive-recurrent Markov process (a continuous-space analog of finite-state irreducible Markov chains), and general theory (cf. Meyn and Tweedie [34] for the discrete-time case) can be used to obtain a central limit theorem, albeit without explicit values of constants.

Proposition 10. For fixed $i \geq 1$, there exists $0<\sigma(i)<\infty$ such that $\sigma\left[S_{n}(i)\right] \sim$ $\sigma(i) \sqrt{\log n}$ and

$$
P\left(\frac{S_{n}(i)-c(i) \log n}{\sigma(i) \sqrt{\log n}} \leq x\right) \rightarrow \Phi(x),-\infty<x<\infty .
$$

5.3. Asymptotics for the central piles. We have seen that the leftmost piles have order $\log n$ cards. Since there are order $n^{1 / 2}$ piles, a typical pile should have order $n^{1 / 2}$ cards. More precisely, there is very good reason to believe that the central pile sizes have a deterministic shape in the limit. Figure 3 illustrates the shape.

Conjecture 11. As $n \rightarrow \infty$ and $i \sim \theta n^{1 / 2}$ for $0<\theta<2$,

$$
\begin{aligned}
E S_{n}(i) & =\left(\theta \log \frac{2}{\theta}\right) n^{1 / 2}+o\left(n^{1 / 2}\right) \\
\sigma^{2}\left[S_{n}(i)\right] & =o\left(n^{1 / 2}\right) .
\end{aligned}
$$




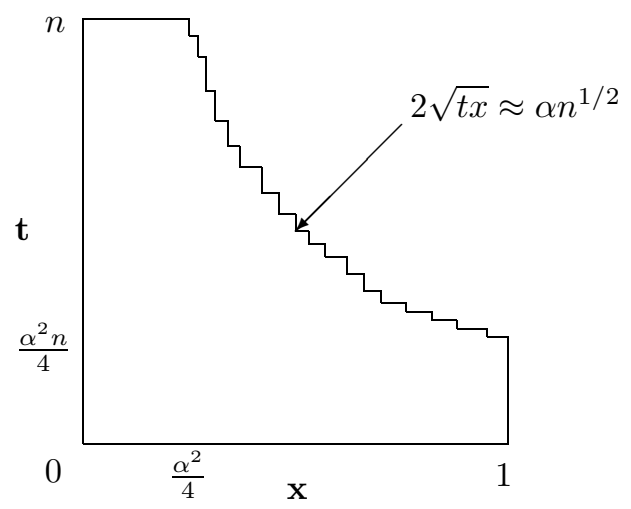

FIGURE 4

What can be easily deduced from known results is an "integrated" version of the assertion of Corollary 11.

Theorem 12. For fixed $0<\alpha<2$, as $n \rightarrow \infty$

$$
\begin{gathered}
E\left(n^{-1} \sum_{i \leq\left\lfloor\alpha n^{1 / 2}\right\rfloor} S_{n}(i)\right) \rightarrow \frac{\alpha^{2}}{4}\left(1+\frac{4}{\alpha^{2}}\right) \\
P\left(\left|n^{-1} \sum_{i \leq\left\lfloor\alpha n^{1 / 2}\right\rfloor} S_{n}(i)-\frac{\alpha^{2}}{4}\left(1+\frac{4}{\alpha^{2}}\right)\right|>\varepsilon\right) \rightarrow 0, \varepsilon>0 .
\end{gathered}
$$

It is not hard to deduce Theorem 12 from the interacting particle proof of $E\left[L_{n}\right] \sim 2 n^{1 / 2}$ outlined in section 3.3. In brief, (8) says that at time $t$ the number of piles whose top card has label less than $x$ is about $2 \sqrt{x t}$. Thus for pile $i=\left\lfloor\alpha n^{1 / 2}\right\rfloor$, the pile appears at about time $\frac{\alpha^{2}}{4} n$, and the label on its top card at subsequent times $t$ is approximately the solution $x$ of $2 \sqrt{x t}=\alpha n^{1 / 2}$. The number of cards at time $n$ in piles to the left of pile $i$ is therefore the number of points of the space-time Poisson process in the region shown in figure 4, which is approximately the area of that region, which works out as $\frac{\alpha^{2}}{4}\left(1+\frac{4}{\alpha^{2}}\right) n$.

Conjecture 11 is the natural "local" counterpart to Theorem 12. If indeed $E S_{n}(i) \sim \beta(\alpha) n^{1 / 2}$ for some constants $\beta(\alpha)$, then using the first assertion of Theorem 12 ,

$$
\beta(\alpha)=\frac{d}{d \alpha} \frac{\alpha^{2}}{4}\left(1+\frac{4}{\alpha^{2}}\right)=\alpha \log \frac{2}{\alpha} .
$$

To prove the conjecture via the probability methods of section 3.3 would involve analysis of the interacting particle system as seen by a tagged particle. Presumably this can be carried out by modifying the arguments used [15] in the context of the simple asymmetric exclusion process.

5.4. Asymptotics for the rightmost pile. Write $R_{n}=S_{L_{n}}(1)$ for the number of cards in the rightmost pile, that is pile $L_{n}$. If we Poissonize, i.e. replace $n$ by an independent Poisson random variable $N(t)$ with mean $t$, then it is not hard to 
deduce the following limit result from the interacting particle proof of $E\left[L_{n}\right] \sim 2 n^{1 / 2}$ outlined in section 3.3.

Theorem 13. As $t \rightarrow \infty$

$$
P\left(R_{N(t)}=r\right) \rightarrow P\left(R_{\infty}=r\right), r=1,2,3, \ldots
$$

where the limit $R_{\infty}$ can be described as follows:

$$
R_{\infty}=R\left(1+\mathcal{P}\left(\xi_{1} \xi_{2}\right)\right)
$$

where $\xi_{1}$ and $\xi_{2}$ are independent with exponential(1) distribution, $\mathcal{P}(\lambda)$ denotes a Poisson r.v. with mean $\lambda$, and $R(m)=S_{m}(1)$ is the number of records in a random permutation of $m$ cards.

Thus if $R_{n}$ converges in distribution, then the limit must be $R_{\infty}$, but we do not see an easy way to exclude the (very implausible) possibility of asymptotic cyclic behavior. Our probabilistic description of $R_{\infty}$ does not translate to a simple explicit formula, but a quick simulation gives

$$
\begin{array}{ccccccc}
r & 1 & 2 & 3 & 4 & 5 & \geq 6 \\
P\left(R_{\infty}=r\right) & 0.744 & 0.189 & 0.050 & 0.013 & 0.003 & 0.001
\end{array}
$$

\section{FinAL REMARKS}

6.1. Other solitaire games. Several books [35], [39] and web sites (accessible via any search engine) are devoted to solitaire. Patience sorting (once one has realized the optimal strategy) is atypical in that no player choice is involved; in this respect it resembles Clock solitaire. In Clock solitaire it is elementary that the chance of winning with an ordinary 52 card deck is 1/13: see Jenkyns and Muller [21] for further elementary analysis.

There has been surprisingly little serious effort to analyze other solitaire games. The most common game (often called just solitaire or patience) is properly called Klondike or Idiot's Delight. This is the game involving placing a card of rank $i$ on a card of rank $i+1$ of opposite color suit, and it is the variant included with Microsoft Windows. Patience sorting could be viewed as a simplification of Klondike. Klondike involves player choice, making it harder to analyze, or even to model via computer simulation. We do not know the chance of winning, or the expected value of the variant in which the player stakes $\$ 52$ and receives $\$ 5$ for each card played forward. Rabb [41] simulated a common form of Klondike in which cards are turned over three at a time (with only the top card exposed) and where one can cycle through the deck indefinitely. She found that the computer won about $8 \%$ of games whereas she won about $15 \%$. In work in progress, Diaconis - Holmes - Koller study modern game-playing heuristics applied to Klondike. Preliminary results suggest a win probability around $15 \%$. Irving Kaplansky (personal communication) reports playing 2000 games of Klondike with the rules above, but also with all cards face up: he had $36.6 \%$ wins. Kuykendall [27] presents a Klondike afficionado's viewpoint on these matters.

Ulam and Mauldin [33] investigate optimal strategies in different examples of "combinatorial games extracted from playing card solitaires." 


\section{ACKNOWLEDGEMENTS}

We thank Bob Floyd and Colin Mallows for discussions of their work, and Mark Nitzberg for the simulations behind figure 3 .

\section{REFERENCES}

[1] D.J. Aldous and P. Diaconis. Hammersley's interacting particle process and longest increasing subsequences. Probab. Th. Rel. Fields, 103:199-213, 1995. MR 96k:60017

[2] B.C. Arnold, N. Balakrishnan, and H.N. Nagarajo. Records. Wiley, 1998. CMP 98:15

[3] J. Baik, P.A. Deift, and K. Johansson. On the distribution of the length of the longest increasing subsequence of random permutations. Technical Report math.CO/9810105, XXX Math Archive, J. Amer. Math. Soc., 12:1119-1178, 1999.

[4] J. Baik, P.A. Deift, and K. Johansson. On the distribution of the length of the second row of a Young diagram under Plancherel measure. Technical Report math.CO/9901118, XXX Math Archive, 1999.

[5] J. Baik and E.M. Rains. Algebraic aspects of increasing subsequences. Technical Report math.CO/9905083, XXX Math Archive, 1999.

[6] J. Baik and E.M. Rains. Generalized increasing subsequence problems II. Asymptotic results. Technical report, Courant Institute, 1999.

[7] P. Billingsley. Convergence of Probability Measures. Wiley, 1968. MR 38:1718

[8] A. Borodin. Longest increasing subsequences of random colored permutations. Technical Report math.CO/9902001, XXX Math Archive, 1999.

[9] A. Borodin, A. Okounkov, and G. Olshanski. On asymptotics of Plancherel measures for symmetric groups. Technical Report math.CO/9905032, XXX Math Archive, 1999.

[10] D. Critchlow. Ulam's metric. In Encyclopedia of Statistical Sciences, volume 9, pages 379-380. Wiley, 1988.

[11] A. De Masi, N. Ianiro, A. Pellegrinotti, and E. Presutti. A survey of the hydrodynamical behavior of many particle systems. In J.L. Lebowitz and E.W. Montrell, editors, Nonequilibrium Phenomena II: From Stochastics to Hydrodynamics, volume 11 of Studies in Statistical Mechanics, pages 123-294. North-Holland, Amsterdam, 1984. MR 86g:82003

[12] R. Durrett. Oriented percolation in two dimensions. The Annals of Probability, 12:999 - 1040, 1984. MR 86g:60117

[13] R. Durrett. Probability: Theory and Examples. Wadsworth, Pacific Grove, CA, 1991. MR 91 m:60002

[14] R. Engelen, P. Tommassen, and W. Vervaat. Ignatov's theorem; a new and short proof. $J$. Appl. Probab., 25A:229-236, 1988. MR 90a:60094

[15] P.A. Ferrari and L.R.G. Fontes. Poissonian approximation for the tagged particle in asymmetric simple exclusion. J. Appl. Probab., 33:411-419, 1996. MR 97j:60183

[16] M.L. Fredman. On computing the length of the longest increasing subsequence. Discrete Math., 11:29-35, 1975. MR 50:6865

[17] I.M. Gessel. Symmetric functions and P-recursiveness. J. Combin. Theory Ser. A, 53:257285, 1990. MR 91c:05190

[18] J.M. Hammersley. First-passage percolation. J. Roy. Statist. Soc. B, 28:491-496, 1966. MR 35:5014

[19] J.M. Hammersley. A few seedlings of research. In Proc. Sixth Berkeley Symp. Math. Statist. and Probability, Volume 1, pages 345-394. University of California Press, 1972. MR 53:9457

[20] G. James and A. Kerber. The Representation Theory of the Symmetric Group. AddisonWesley, Reading, MA, 1981. MR 83k:20003

[21] T.A. Jenkyns and E.R. Muller. A probabilistic analysis of clock solitaire. Math. Magazine, 54:202-208, 1981. MR 83h:60015

[22] K. Johansson. Shape fluctuations and random matrices. Technical report, Royal Inst. Technology, Stockholm, 1999.

[23] N. L. Johnson and S. Kotz. Continuous Univariate Distributions, volume 2. Wiley, 1970. MR 96j:62029

[24] S.V. Kerov and A.M. Vershik. Asymptotic theory of the characters of the symmetric group. Functional Anal. Appl., 15:246-255, 1981. MR 84a:22016

[25] H. Kesten. On the speed of convergence in first-passage percolation. Ann. Appl. Probab., 3:296-338, 1993. MR 94m:60205 
[26] J. H. Kim. On increasing subsequences of random permutations. J. Combin. Theory Ser. A, 76:148-155, 1996. MR 97f:60024

[27] C. Kuykendall. Analyzing solitaire. Science, 283(5403):794-795, Feb. 5, 1999.

[28] T.M. Liggett. Interacting Particle Systems. Springer-Verlag, 1985. MR 86e:60089

[29] B.F. Logan and L.A. Shepp. A variational problem for random Young tableaux. Advances in Math., 26:206-222, 1977. MR 98e:05108

[30] I. MacDonald. Symmetric Functions and Hall Polynomials. Oxford University Press, 1979. MR 84g:05003

[31] C.L. Mallows. Problem 62-2, patience sorting. SIAM Review, 5:375-376, 1963.

[32] C.L. Mallows. Patience sorting. Bull. Inst. Math. Appl., 9:216-224, 1973.

[33] R. D. Mauldin and S.M. Ulam. Mathematical problems and games. Adv. Appl. Math., 8:281344, 1987. MR 89g:04005

[34] S.P. Meyn and R.L. Tweedie. Markov Chains and Stochastic Stability. Springer-Verlag, 1993. MR 95j:60103

[35] Albert H. Morehead and Geoffrey Mott-Smith. The Complete Book of Solitaire and Patience Games. Bantam, 1973.

[36] A.M. Odlyzko, B. Poonen, H. Widom, and H. Wilf. On the distribution of longest increasing subsequences in random permutations. In Preparation, 1998.

[37] A.M. Odlyzko and E.M. Rains. On longest increasing subsequences in random permutations. Technical report, AT\&T Labs, 1998.

[38] A. Okounkov. Random matrices and random permutations. Technical Report math.CO/9903176, XXX Math Archive, 1999.

[39] David Partlett. Solitaire: Aces Up and 399 Other Card Games. Pantheon, 1979.

[40] S. Pilpel. Descending subsequences of random permutations. J. Combinatorial Th. A, 53:96116, 1990. MR 91d:05001

[41] A. Rabb. A probabilistic analysis of the game of solitaire, 1989. Undergraduate Honors Thesis, Harvard.

[42] E. M. Rains. Increasing subsequences and the classical groups. Electron. J. Combinatorics, 5:R12, 1998. MR 98k:05146

[43] H. Rost. Nonequilibrium behavior of a many particle process: Density profile and local equilibrium. Z. Wahrsch. Verw. Gebiete, 58:41-53, 1981. MR 83a:60176

[44] B.E. Sagan. The Symmetric Group: Representations, Combinatorial Algorithms and Symmetric Functions. Wadsworth, 1991. MR 93f:05102

[45] C. Schensted. Longest increasing and decreasing subsequences. Canad. J. Math., 13:179-191, 1961. MR 22:12047

[46] T. Seppäläinen. Hydrodynamic scaling, convex duality and asymptotic shapes of growth models. Markov Process. Related Fields, 4:1-26, 1998. MR 99e:60221

[47] R. Stanley. Enumerative Combinatorics, Vol. 2. Cambridge University Press, 1999. CMP 99:09

[48] R. P. Stanley. Enumerative Combinatorics, Vol I. Wadsworth \& Brooks/Cole, Monterey, California, 1986. MR 87j:05003

[49] D. Stanton and D. White. Constructive Combinatorics. Undergraduate Texts in Mathematics. Springer-Verlag, 1986. MR 88a:05001

[50] C.A. Tracy and H. Widom. Level-spacing distributions and the Airy kernel. Comm. Math. Phys., 159:151-174, 1994. MR 95e:82003

[51] C.A. Tracy and H. Widom. Random unitary matrices, permutations and Painlevé. Technical Report math.CO/9811154, XXX Math Archive, 1998.

[52] C.A. Tracy and H. Widom. On the distribution of the lengths of the longest monotone subsequences in random words. Technical Report math.CO/9904042, XXX Math Archive, 1999.

[53] S.M. Ulam. Some ideas and prospects in biomathematics. Ann. Rev. Biophys. Bioeng., 1:277$292,1972$.

[54] R. Venkatsubramani. Hydrodynamic Limit for the Asymmetric Exclusion Process with Deterministic Initial Data and the Hammersley Process on $S^{1}$. Ph.D. thesis, New York University, 1995.

[55] A.M. Vershik and S.V. Kerov. Asymptotics of the Plancherel measure of the symmetric group and the limiting form of Young tables. Soviet Math. Dokl., 18:527-531, 1977. Translation of Dokl. Acad. Nauk. SSSR 233 (1977) 1024-1027. 
[56] A.M. Vershik and S.V. Kerov. Asymptotic behavior of the maximum and generic dimensions of irreducible representations of the symmetric group. Functional Anal. Appl., 19:21-31, 1985. MR 85k: 11051

[57] M.S. Waterman and M. Vingron. Sequence comparison significance and Poisson approximation. Statist. Science, 9:367-381, 1994. CMP 95:10

Department of Statistics, 367 Evans Hall, University of California, Berkeley, CA 94720

E-mail address: aldous@stat.berkeley.edu

Departments of Mathematics and Statistics, Stanford University, Stanford, CA 94305 